SCIENTIFIC LETTER

\title{
Left superior caval vein: a powerful indicator of fetal coarctation
}

\author{
L Pasquini, A Fichera, T Tan, S Y Ho, H Gardiner
}

Heart 2005;91:539-540. doi: 10.1136/hrt.2004.045443

A persistent left superior caval vein (LSVC) is present in about $0.3 \%$ of the population at necropsy and in adults undergoing pacing. ${ }^{1}$ Although it may occur in up to $9 \%$ of necropsies of children with congenital heart disease $(\mathrm{CHD}),{ }^{2}$ its prevalence in the fetus at increased risk of, or with, CHD has not yet been determined. We document the incidence, associations, and outcome of LSVC in 1678 consecutive fetuses referred for echocardiography to a fetal medicine unit between January 1997 and December 2002.

\section{METHODS}

Fetal echocardiograms were performed (Acuson Sequoia C256/Siemens Medical Solutions, Acuson Ultrasound Department, Mountain View, California) using a 6C2, 8C4 or 5V2c probe and persistence of the LSVC documented from routine views of the four chambers and transverse arch (the three vessel view). Postnatal diagnosis was confirmed by echocardiogram, at surgery, or at necropsy.

The odds ratio (95\% confidence intervals (CI)) for CHD in the presence of LSVC and the odds ratio of an associated cardiac defect being coarctation of the aorta (CoA) were calculated.

\section{RESULTS}

Of 1678 cardiac referrals, 1230 were normal with an LSVC detected in four patients $(0.3 \%)$. Cardiac abnormality was present in 448 cases $(27 \%)$, with persistent LSVC in 12 cases $(2.7 \%)$. Thus, the odds ratio of a fetus having a cardiac defect if a LSVC is detected is 8.43 (95\% CI 2.71 to 26.29 ).
Table 1 details the cardiac diagnosis, associations and outcomes of the 16 fetuses with persistent LSVC. It was associated with situs inversus in one fetus, and five fetuses showed interruption of the inferior vena cava (IVC) with azygous/hemiazygous continuation to a persistent LSVC which drained to the coronary sinus in all but one fetus. All five fetuses had the usual arrangement of the atrial appendages, confirmed postnatally. ${ }^{3}$ LSVC was seen in five of the 10 fetuses with aortic arch hypoplasia (50\%), nine of whom required surgery for CoA, and one with Turner syndrome and arch hypoplasia was managed conservatively. The odds ratio of the cardiac defect being a CoA in fetuses with LSVC is 61.57 (95\% CI 14.48 to 261.74 ).

\section{DISCUSSION}

This study has documented persistent LSVC in $1 \%$ of a consecutive series of fetuses selected for detailed echocardiography. While the $0.3 \%$ prevalence of LSVC in those with structurally normal hearts was similar to previous population studies, LSVC was associated with an eightfold increase in CHD in this fetal population, and strongly associated with CoA.

The prevalence of LSVC in the fetal population remains uncertain. The importance of the three vessel view, where the LSVC is most easily identified, is only now becoming recognised by obstetric sonographers as part of complete fetal screening. However, fetuses with LSVC may be referred with four chamber disproportion caused by an enlarged coronary sinus, or as suspected atrioventricular or atrial septal defect. Furthermore, in our series $25 \%$ had important

Table 1 Cardiac diagnosis, extracardiac malformations, karyotype, and outcome in 16 fetuses with left superior caval vein

\begin{tabular}{|c|c|c|c|c|c|}
\hline Case & Fetal cardiac diagnosis & Situs & Karyotype & Extracardiac malformations & Outcome \\
\hline $\mathrm{CHD}$ & S,C,C, unbalanced AVSD, dysplastic LAVV & $\mathrm{N}$ & $46 X Y$ & Soft marker (two vessel cord) & Surgery, well \\
\hline CHD & $S, C, C$, sub-Ao VSD, CoA & $\mathrm{N}$ & $46 X Y$ & Renal agenesis, SGA, ventriculomegaly, talipes & NND \\
\hline CHD & S,C,C, CoA (postnatal hypoplasia only), PE & $\mathrm{N}$ & $45 \times 0$ & None & Well \\
\hline $\mathrm{CHD}$ & $\mathrm{S}, \mathrm{C}, \mathrm{C}$, muscular VSD, CoA & $\mathrm{N}$ & $46 X X$ & None & Surgery, well \\
\hline $\mathrm{CHD}$ & S,C,C, PMVSD, CoA & PV & $46 X Y$ & $\begin{array}{l}\text { SGA, postnatal malrotation and biliary atresia, } \\
\text { right sided stomach }\end{array}$ & Surgery, well \\
\hline $\mathrm{CHD}$ & $S, C, C, C o A$ & PV & $46 \times X$ & None & Surgery, well \\
\hline CHD & $S, C, D$, large VSD, PS, criss-cross heart & PV & $46 X Y$ & Soft marker (mild hydronephrosis) & Surgery, well \\
\hline $\mathrm{CHD}$ & SI,C, DORV, restrictive VSD, straddling MV & $\mathrm{SI}$ & $46 X Y$ & None & Postoperative death \\
\hline CHD & $S, C, C$, infradiaphragmatic TAPVC & PV & $46 X Y$ & Massive bilateral pulmonary sequestration & NND \\
\hline CHD & $S, C, C, V S D$ and TAPVC to confluence & $\mathrm{N}$ & T18 & Two vessel cord, SGA, rocker-bottom feet, microcephaly & NND \\
\hline CHD & $S, C, C$, TOF & $N$ & $46 X Y$ & None & Surgery, well \\
\hline $\mathrm{CHD}$ & $\begin{array}{l}\text { S,C,C, dextrocardia, unbal.AVSD, } \\
\text { aortic atresia }\end{array}$ & PV & $46 X Y$ & None & NND \\
\hline Normal & $S, C, C$, right aortic arch & $\mathrm{N}$ & $46 X X$ & None & Well \\
\hline Normal & $S, C, C$, small $P E$ & $\mathrm{~N}$ & $46 X X$ & None & Well \\
\hline Normal & $S, C, C$, biventricular hypertrophy & $\mathrm{N}$ & $46 X X$ & None & Well \\
\hline Normal & $S, C, C$ & $\mathrm{~N}$ & $45 \times 0$ & None & Well \\
\hline
\end{tabular}

45X0, Turner syndrome; AVSD, atrioventricular septal defect; C, concordant atrioventricular or ventriculoarterial connection; CHD, congenital heart disease; CoA, coarctation of the aorta; D, discordant atrioventricular or ventriculoarterial connection; DORV, double outlet right ventricle; LAVV, left atrioventricular valve; N, normal situs; NND, neonatal death without cardiac surgery; PE, pericardial effusion; PMVSD, perimembranous ventricular septal defect; PV, posterior vein (azygous/hemiazygous) and absent inferior vena cava at situs; S, situs solitus; SGA, small for gestational age; SI, situs inversus; T18, trisomy 18; TAPVC, total anomalous pulmonary venous connections; TOF, tetralogy of Fallot; unbal, unbalanced; VSD, ventricular septal defect. 
extracardiac malformations and three had aneuploidy that may have been detected sonographically.

The prevalence of LSVC in $2.7 \%$ of fetuses with CHD is less than the $9 \%$ reported from pathological series where atrioventricular septal defect, cor triatriatum, and mitral atresia were common associations. ${ }^{2}$ However, there was a $6 \%$ prevalence of LSVC in the 107 fetuses in our cohort with serious malformations associated with ventricular or arterial disproportion. Of particular importance was the association with CoA or arch hypoplasia. This lesion remains the one with most diagnostic uncertainty in fetal life and in common with others, we found an $89 \%$ specificity of diagnosis before 24 weeks of gestation but only $17 \%$ after. ${ }^{4}$

Cardiac growth velocities are well documented in the fetus ${ }^{5}$ but early growth may differ in those with LSVC. Necropsy associations with cor triatriatum and mitral atresia suggest that the mechanism responsible for failure to close the LSVC may be associated with abnormal left sided connections or abnormal early flow patterns that reduce left ventricular filling and may explain in part the association with CoA, unbalanced atrioventricular septal defect, and total anomalous pulmonary venous connections reported here. Serial scans in this series suggest that it exerts less haemodynamic influence later in gestation, and left sided growth proved adequate for a biventricular repair in all cases where surgery was necessary, with important implications for first trimester counselling.

This study suggests that sonographic detection of LSVC is a powerful indicator of fetuses with probable CHD in whom CoA was the most common diagnosis. LSVC is easily recognised on standard cardiac screening views and we recommend its detection should prompt referral for detailed echocardiography.

\section{ACKNOWLEDGEMENTS}

LP is supported by echo UK, the fetal heart charity (www.echocharity. org.uk).

\section{Authors' affiliations}

L Pasquini, A Fichera, T Tan, H Gardiner *, Faculty of Medicine, Imperial College London, Queen Charlotte's and Chelsea Hospital, London, UK

S Y Ho, Faculty of Medicine, Imperial College London, Royal Brompton Hospitals, London UK

*Also at Faculty of Medicine, Imperial College London, Royal Brompton Hospitals, London, UK

Correspondence to: Dr Helena M Gardiner, Institute of Reproductive and Developmental Biology, Faculty of Medicine, Imperial College, Sydney Street, London SW3 6NP, UK; helena.gardiner@imperial.ac.uk

Accepted 14 August 2004

\section{REFERENCES}

1 Biffi M, Boriani G, Frabetti L, et al. Left superior vena cava persistence in patients undergoing pacemaker or cardioverter-defibrillator implantation. Chest 2001:120:139-44.

2 Nsah EN, Moore GW, Hutchins GM. Pathogenesis of persistent left superior vena cava with a coronary sinus connection. Pediatr Pathol 1991;11:261-9.

3 Pasquini L, Tan T, Ho SY, et al. Abnormal arrangement of abdominal vessels: implications for fetal outcome? Cardiol Young (in press).

4 Sharland GK, Chan KY, Allan LD. Coarctation of the aorta: difficulties in prenatal diagnosis. Br Heart J 1994;71:70-5.

5 Hornberger LK, Sanders SP, Rein AJJ, et al. Left heart obstructive lesions and left ventricular growth in the mid-trimester fetus. Circulation 1995;92:1531-8.

\section{IMAGES IN CARDIOLOGY}

\section{Quadrant pulmonary oedema}

A 73 year old man presented to the emergency department with a three day history of dyspnoea. He had no past medical history. On admission his blood pressure was $120 / 80 \mathrm{mmHg}$, heart rate $120 \mathrm{bpm}$, and respiratory rate 24 breaths per minute. On auscultation inspiratory crackles were heard over the mid and upper portion of the right hemithorax along with a minor systolic heart murmur.

Apart from sinus tachycardia, the ECG was unremarkable. An infiltrate in the upper lobe of the right lung was seen on a standard chest $x$ ray. The echocardiogram showed a partially flailed posterior mitral leaflet caused by ruptured chordae tendinae with severe, eccentric regurgitation directed towards the right upper pulmonary vein. The pulmonary capillary wedge pressure (PCWP) in the right upper lobe exceeded the PCWP of the left lung by $20 \mathrm{~mm} \mathrm{Hg}$.

We have described a rare case of unilateral pulmonary oedema, restricted to the right upper lung lobe (also termed quadrant pulmonary oedema), which was caused by acute, eccentric mitral regurgitation towards the right upper pulmonary vein. After mitral valve repair the chest $x$ ray was normal.

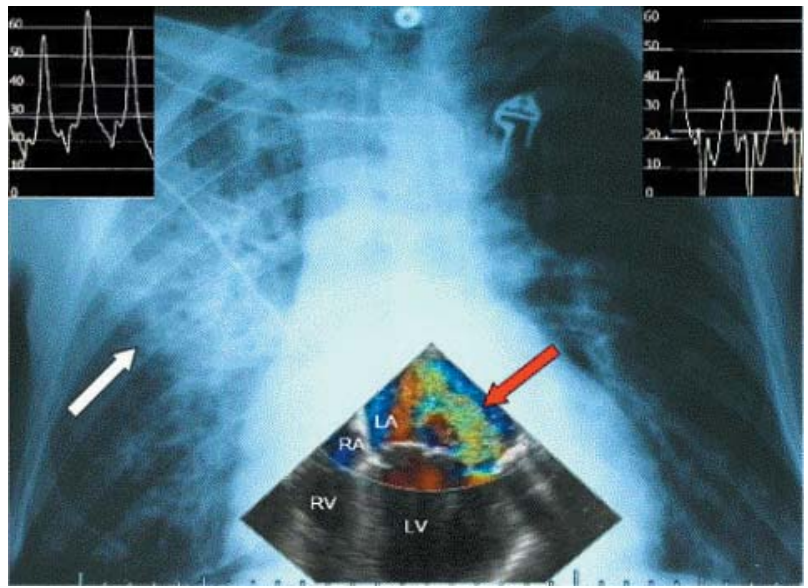

Standard chest $x$ ray with a right upper lobe infiltrate (white arrow). Transoesophageal echocardiography still frame: mitral valve regurgitant jet directed towards the right upper pulmonary vein (red arrow). LA, left atrium; LV, left ventricle; RA, right atrium; RV, right ventricle. Left PCWP tracing with $v$ wave of $40 \mathrm{~mm} \mathrm{Hg}$ (right upper corner) versus $v$ wave of $60 \mathrm{~mm} \mathrm{Hg}$ in the right PCWP tracing (left upper corner).
J D Keyser

K Dujardin

W Mullens

wmulle0@hotmail.com 